\title{
Towards a Comprehensive Blockchain Architecture Continuum
}

\author{
Leonardo Maria De Rossi \\ SDA Bocconi School of \\ Management - DEVO Lab \\ $\underline{\text { leonardo.derossi@,unibocconi.it }}$
}

\author{
Gianluca Salviotti \\ SDA Bocconi School of \\ Management - DEVO Lab \\ gianluca.salviotti@s,sdabocconi.it
}

\author{
Nico Abbatemarco \\ SDA Bocconi School of \\ Management - DEVO Lab \\ nico.abbatemarco@sdabocconi.it
}

\begin{abstract}
The increasingly need for companies to keep a high level of synchronization globally and the advent of new technologies are pushing more and more to move decision-making and operational power from the centre of organizations to their edges. The blockchain could be the key technology to make this change possible.

However, there is no bridge yet capable of shortening the still long distance between this new technological phenomenon and today's business realities. Our work aims precisely at this goal; we propose a framework of blockchain models to help practitioners understanding and potentially implement new solutions based on this technological paradigm.

In particular, we have developed an ontology that helps to identify and clarify in detail what are the concepts and structures revolving around this technology, and built a continuum of blockchain architectural solutions, ranging from a classic centralized IT architecture to one completely distributed within a public ecosystem.
\end{abstract}

\section{Background}

Beyond the media clamour aroused by cryptocurrencies in 2017, the technology underlying these digital assets - namely the blockchain - has gained its own popularity within the business community during the last two years. McKinsey reports that 2016 and 2017 alone have seen more than half a million new publications on and 3.7 million Google search results for blockchain [1].

Blockchain has also been one of the key disruptive technologies discussed at Davos during the last World Economic Forum. Experts involved in the main roundtable stated that "many big financial institutions are investing plenty of time and money exploring the potential of blockchain technology to improve a range of activities from post-trade settlement in financial markets and cross-border payments to trade finance and syndicated loans" [2].
Following this path, big players far from financial services - such as Walmart and Maersk - also started their blockchain journeys, with the aim to test the benefits of distributed ledger technologies across complex supply chains [3]. Supply chain management seems to be one of the most promising application fields for business-oriented blockchains [4,5]. Apart from cryptocurrencies, financial transactions and supply chain management, other interesting applications of blockchain technologies are related to advanced IoT tracking, cloud storage, digital identity, energy management and distribution [6].

Most tellingly, large investments in blockchain are being made. The overall Venture Capital funding for blockchain-focused start-ups was up to $\$ 1.5$ billion in 2016 [7]. Leading technology players - such as IBM and Microsoft - are also heavily betting on blockchain. The goal is to make available cloud-platforms that could be easily accessed by client organizations in order to build decentralized applications [8].

Unfortunately, the result of this broad enthusiasm has led to a hype effect around blockchain [9,10,11]. While it is commonly accepted that blockchain could drive radical changes in many industries [12], with a potential impact on the whole economy $[1,13]$, several authors focus on the medium-to-long time needed in order to actually experience some transformational impacts of this technology.

From a merely enterprise perspective, during the same World Economic Forum roundtable mentioned above, while talking about financial institutions trying to cope with blockchain implementations, experts recognized that "because blockchain's benefits come from decentralisation there is little point replacing one technology with another without changing the business model" [2].

According to the insights collected by practitioneroriented institutions, too many executives and IT managers are launching projects based solely on the hype around the technology [14]. This leads to a series of common mistakes [15], such as:

- misunderstanding or ignoring the purpose of blockchain technology;

- assuming that current technology is ready for production use; 
- viewing blockchain technology purely as a database or storage mechanism;

- ignoring funding and governance issues for a peerto-peer distributed network.

Moreover, the complete lack and/or inadequacy of blockchain standards in terms of governance models, enterprise-grade security, legal, tax and accounting frameworks, native interoperability and scalability makes addressing business challenges and opportunities beyond the original use case impractical and/or risky $[16,17]$.

The combined effect of these empirical evidences seems to be slowing down the development of blockchain and will rapidly lead to a post-hype status [18]. Furthermore, the level of intrinsic complexity of the technology is not facilitated its diffusion at the company level. From a technical perspective, a blockchain could be viewed as a multi-layered architecture that entails a wide set of elements related to data structure, networks, advanced cryptography, consensus mechanisms and so on.

A great gap is still present between the technology and its adoption by companies and a complete study of all the business and governance models that can be realized thanks to different implementation of the blockchain is still lacking. According to Morabito [19] the free nature of existing distributed networks calls into question factors such as digital divide, unaccountable power of core developers, and lack of clearness in decision making process, thus making some blockchain advocates' expectations overestimated and unrealistic. Iansiti and Lakhani [11] state that "it will take decades for blockchain to seep into our economic and social infrastructure. [...] Many barriers - technological, governance, organizational, and even societal - will have to fall."

Consistently with the described background, our hypothesis is that, at a corporate level, these barriers tend to emerge when there is a mismatch between the configuration of the technical components of the blockchain and their related business and governance layers. In Nakamoto's original white paper, the blockchain was intended as a tool to disrupt and disintermediate centralized entities (e.g. financial institutions). On the contrary, many recent implementations of the technology are limited to an update of the existing centralized architectures. According to Gartner [18], in this situation, "technical and business silos are reinforced, business models are preserved, architecture made more complex, the potential for vendor lock-in encouraged and the potential creation of new value structures are inhibited".
Starting from these considerations, our work aims at addressing the following questions:

- Which is the current role for fully centralized blockchain architectures?

- Could they be the starting point to create a more sophisticated business and organizational approach to the original concept of blockchain?

- Which are the alignment variables addressed in the currently implemented/experimented blockchain architectures?

- Is there a continuum of architectural options that a company should leverage in order to design its own way to implement a blockchain and get value from $i t$ ?

Leveraging on the existing literature on the topic, in particular with regard to what has been said about the ontology of the blockchain and the taxonomic and semantic classifications proposed by previous authors $[10,20,21,22]$, we propose a comprehensive architecture continuum entailing all the relevant combinations of technical and business options currently available to implement a blockchain solution. The continuum starts by proposing a completely private solution, theoretically closer to the existing IT models, to move progressively towards a full public solution. Using case studies with a theory building approach [23], the paper provides a full description of each architectural model, focusing on both technical and business/governance variables.

The work is structured as follows. In the next section, we will present a brief literature review on this new technology and a quick explanation of all the terminology that will be used in the rest of the work. We will then be able to build a proposal of ontology useful to analyse blockchain architectures and their alignment options. In the methodology paragraph, we will explain the design of our research, whose outputs will then be examined in the continuum analysis section. Finally, a critical review of our work will be presented proposing some future outlooks in order to connect this academic work to the real business landscape.

\section{Literature Overview}

\subsection{Key features of a blockchain}

A blockchain can be defined as a decentralized database structured in blocks, each one containing a certain amount of information and distributed through a chain (i.e. a ledger) over a network. Hence, it is a digital way to store any kind of data through a network $[19,24]$. 
In particular, a blockchain is a distributed network, meaning that data contained in the ledger are constantly shared and synchronized across its participants, even if they are spread across multiple sites, institutions or geographies. Each participant in the network can access the recordings shared across the ledger and replicate them. If compliant to the rules established in the protocol and confirmed by the network, any addition made to the ledger is automatically mirrored in all of its copies [25].

The keystone of the entire blockchain technology is the so-called consensus mechanism, that ensures that the information entered in the blocks are correct and consistent with the rules established in the protocol [26]. Practically, it allows information to be shared between two participants belonging to the network without necessarily having to transit for a central entity to validate the content first $[12,27,28]$.

\subsection{Blockchain architectural layers}

A first step to undertake in order to understand the business and organizational impacts [29] blockchain application is the comprehension of its IT architecture.

Blockchain can be considered as an IT architecture composed by three layers:

- Top layer - Blockchain application. The final service developed by the company using the blockchain;

- Middle layer - Blockchain ledger. The distributed ledger on which the blockchain application is built;

- Bottom layer - Blockchain hardware/network. The network is represented by the sum of all the nodes making use of their computational power to participate in the consensus mechanism confirming or rejecting new transactions - and to store the whole history of the transactions ever occurred in that specific blockchain.

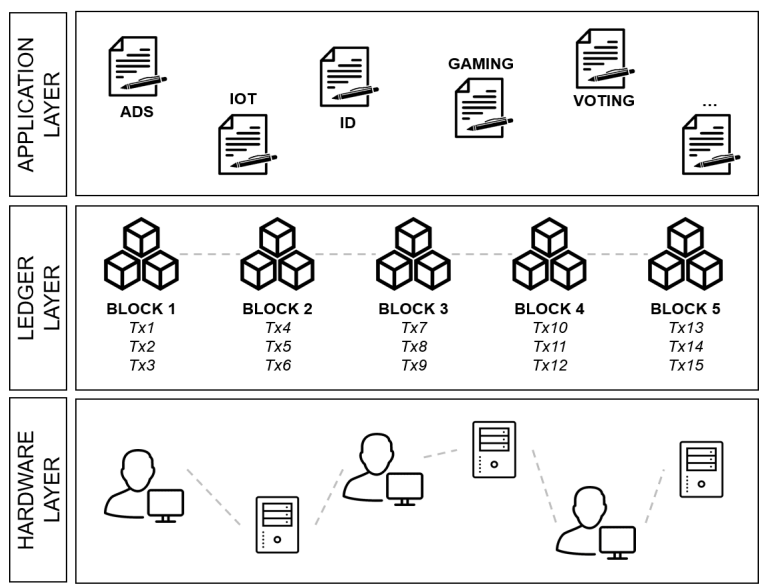

Figure 1. Blockchain as an IT architecture

\subsection{Blockchain governance models}

A blockchain governance model can be classified within two dimensions: "permissioned/permissionless" and "public/private" [30]. The first dimension refers to the ability to take part in the consensus mechanism while the second one is related to the possibility for a user to access the proper blockchain application. More specifically:

- In a permissionless blockchains anyone, including malicious actors, can participate in the consensus process. Thus, anyone is free to be an active part of the network. Costs are higher, and speed is slower than on a permissioned chain;

- Permissioned blockchains are kept centralized to one (or more) authorized user. In this case, the authorized user(s) verifies each transaction. Read permissions may be public or restricted to an arbitrary extent.

On the other hand, both permissioned and permissionless blockchain can be either public or private.

- In a public ecosystem, anyone can join the network and use the application enabled by the blockchain technology. A user can access a specific service without the authorization of the service provider. In a public permissionless blockchain, there is no central authority and everyone with an internet connection can use the service (write), read the transaction's history (read) and, eventually, participate to the consensus mechanism (commit). For example, Bitcoin is a public ecosystem in which every person is able to send/receive a transaction;

- In a private blockchain the final users are known and vetted, and they will be able to access the blockchain service only if the service provider allows them to. Thereby, participants are held accountable through legal terms and conditions (outside of blockchain) and are incentivised to behave honestly to avoid legal prosecution or repercussion. For example, the smart contracts of a decentralized application written on Ethereum would be readable by anyone, but the access to the application itself could be restricted by its developers.

On the basis of the aforementioned dimensions, it is possible to identify four types of blockchain governance models. 
Table 1. Types of blockchains by ownership

\begin{tabular}{|c|l|l|l|l|}
\hline & & Read & Write & Commit \\
\hline \multirow{2}{*}{$\Xi$} & $\begin{array}{l}\text { Public } \\
\text { Permissionless }\end{array}$ & $\begin{array}{l}\text { Open to } \\
\text { anyone }\end{array}$ & Anyone & Anyone \\
\cline { 2 - 5 } & $\begin{array}{l}\text { Private } \\
\text { Permissionless }\end{array}$ & $\begin{array}{l}\text { Open to } \\
\text { anyone }\end{array}$ & $\begin{array}{l}\text { Authorized } \\
\text { participants }\end{array}$ & Anyone \\
\hline \multirow{2}{*}{$\begin{array}{l}\text { aublic } \\
\text { Permissioned }\end{array}$} & Restricted & Anyone & $\begin{array}{l}\text { Authorized } \\
\text { participants }\end{array}$ \\
\cline { 2 - 5 } $\bar{\Xi}$ & $\begin{array}{l}\text { Private } \\
\text { Permissioned }\end{array}$ & Restricted & $\begin{array}{l}\text { Network } \\
\text { only }\end{array}$ & $\begin{array}{l}\text { Network } \\
\text { only }\end{array}$ \\
\hline
\end{tabular}

\section{An ontology to understand blockchain architectural options}

Despite the enthusiasm shown by exponents of the international academic community and of various industries, especially in the financial sector, the studies related to the topic have often been framed in a monothematic way (e.g. analysing only economical or technical aspects such as the mechanism consent or scalability of a protocol). There are only a few multidisciplinary studies that can help the political and economic community to appreciate the benefits brought by the blockchain.

From this point of view, developing a complete ontology regarding the blockchain technology could represent a considerable leap forward, with regard to the general understanding of the topic and its diffusion.

This is exactly the theme covered in the blockchain ontology by Glaser [20], which refers in turn to what was proposed in previous years regarding IT theories. Ontologies can provide a framework for structured knowledge representation, helping establishing concepts and their relationships in a specific domain context. Different variations of this type of theory are schemata, conceptual frameworks and taxonomies.

In his ontology, Glaser subdivides blockchain protocols in a matrix, classifying them accordingly to their independence level on one side and on their operating layers (hardware layer, fabric layer, and application layer) on the other.

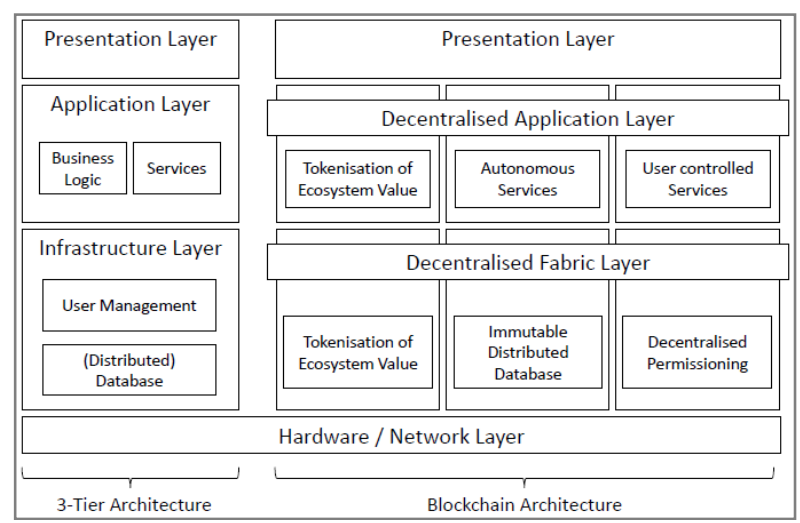

Figure 2. Layers of a blockchain system
Referring to the same ontology, Notheisen et al. [10] developed in 2017 another layer-based representation of blockchain, adding two more layers to the representation formulated by Glaser: the agent and the environment layers. In their work, they affirm that "in combination with the environment layer, the agent layer allows the analyses of market outcomes, application performance, or other system properties from a macroeconomic perspective, and the study of the individual's behaviour from a microeconomic perspective".

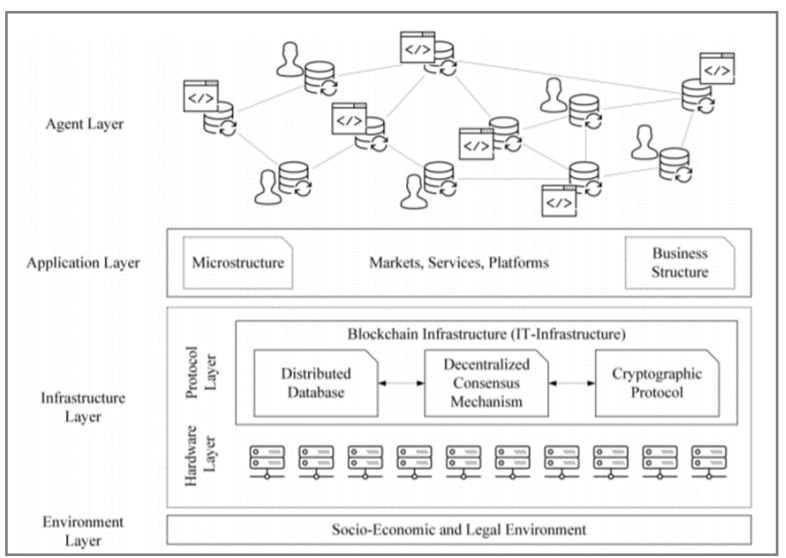

Figure 3. Blockchain market engineering framework

As already stated in 1999 by Benbasat and Zmud [31], theoretical research works, especially in the fields of the Information Systems, often represent an end in themselves instead of being aimed at bringing a greater knowledge, practically relevant, of certain topic of interest. On the contrary, action research pieces, such as articles that address enduring (or current) organizational problems, challenges, and dilemmas as well as articles that address timely business issues, tend to be regularly appreciated by practice, as they help solving current practical problems while expanding scientific knowledge [32,33]. Our work points exactly in this direction. The aim is to realize an ontology to understand blockchain architectural options that conciliates a scientifically rigorous and solid theoretical approach and, at the same time, to keep such tool easy to understand and applicable by a practitioner to make relevant implementation decisions in a real business context.

The word "ontology" is used with reference to what expressed by Uschold and Gruninger [34], i.e. as a common language useful to facilitate an effective communication between people and organizations when talking about the nature and the components of a new theory. With reference to Gregor [35], our 
ontology can either be framed as an analyticaldescriptive theory, as it provides a reference descriptive framework for the blockchain phenomenon and the constructs connected to it, or as a design/action theory, as it offers all the elements necessary for the theoretical construction of an artifact based on this new technology.

In our work, we deal with the concept of blockchain architecture, with architecture being defined as the conceptual and logical structure of a functional system [36,37]. Firstly, on the basis of the above analysed academic body of knowledge, we describe its basic technical elements and define its characteristics and purposes, embracing the current findings of the literature in terms of structure components and layers.

Table 2 - Blockchain technical aspects

\begin{tabular}{|l|l|l|}
\hline Dimension & Variable & Description \\
\hline \multirow{4}{*}{ Technical } & Application layer & $\begin{array}{l}\text { The piece of code developed } \\
\text { to interact with the ledger }\end{array}$ \\
\cline { 2 - 3 } & $\begin{array}{l}\text { Ledger (or Fabric) } \\
\text { layer }\end{array}$ & $\begin{array}{l}\text { The ledger exploited by the } \\
\text { application }\end{array}$ \\
\cline { 2 - 3 } & $\begin{array}{l}\text { Hardware/Network } \\
\text { layer }\end{array}$ & $\begin{array}{l}\text { The hardware deployed by } \\
\text { the network }\end{array}$ \\
\hline
\end{tabular}

Then, in order to get a dynamic view of all the existing options available for a blockchain implementation within an enterprise landscape, we moved forward, adding two further dimensions to go beyond the currently existing models and above all to allow practitioners to have a holistic vision of how all five dimensions can be configured according to a company needs and specific business models.

Table 3. Blockchain governance/business aspects

\begin{tabular}{|l|l|l|}
\hline Dimension & Variable & Description \\
\hline Governance & $\begin{array}{l}\text { Type } \\
\text { ownership and } \\
\text { governance }\end{array}$ & $\begin{array}{l}\text { The governance and } \\
\text { ownership models of the } \\
\text { considered solution }\end{array}$ \\
\hline Business & Revenue model & $\begin{array}{l}\text { The impact of the } \\
\text { blockchain solution in terms } \\
\text { of returns distributed to the } \\
\text { stakeholders }\end{array}$ \\
\hline
\end{tabular}

\section{Research methodology}

To test our ontology and understand the main features of the blockchain architectural models currently implemented, we have adopted a qualitative approach based on a case study research methodology. We felt that this type of approach was ideal to explain the deeply social implications of a technology such as blockchain, to avoid a meaningful loss in terms of comprehension of its public and institutional context as often happens when trying to quantify textual data
[38]. As also stated by Myers [39] "Qualitative research methods are designed to help researchers understand people and the social and cultural contexts within which they live."

The construction of theories starting from use cases is of crucial importance in a field such as that of information systems, given that the study of IS within enterprises has become over time more and more an analysis of the organizational issues revolving around a new technology rather than the technical ones [40].

Firstly, we interviewed the biggest international players currently operating on blockchain technology. Following their reports, we collected a total of 85 descriptions of blockchain enterprise solutions. Therefore, we selected among these the projects that already overcame a concept investigation phase, as defined in [41], eventually selecting 19 solutions.

We decided to select few but consistent specific cases capable of effectively "pattern-match" theory and data [42]: the comparison between multiple use cases allowed the construction of a more robust theory [43], in addition to better delineating the type of relationships existing between the various models [42] and setting an appropriate level of abstraction [44].

Table 4. Selected use cases

\begin{tabular}{|l|c|}
\hline Blockchain solution & $\begin{array}{c}\text { Production } \\
\text { Phase }\end{array}$ \\
\hline The vinicultural supply chain in Italy & Ramp-up \\
\hline IoTappo & Prototype \\
\hline Acincoin & Prototype \\
\hline Enerchain & Pilot \\
\hline $\begin{array}{l}\text { Maersk: cross-border supply chain } \\
\text { solution }\end{array}$ & Pilot \\
\hline $\begin{array}{l}\text { Hejia: blockchain for pharma } \\
\text { procurement }\end{array}$ & Pilot \\
\hline Walmart: blockchain for pork meat & Prototype \\
\hline $\begin{array}{l}\text { 4Trace: a blockhain traceability } \\
\text { platform }\end{array}$ & Prototype \\
\hline SAP - ATB Bank \& Reise Bank AG & Prototype \\
\hline Timestamping Intesa Sanpaolo & Pilot \\
\hline That's Mine & Pilot \\
\hline Ballotchain & Pilot \\
\hline Smartbond & Basic Design \\
\hline Notarchain & Pilot \\
\hline Insurechain & Pilot \\
\hline Securechain & Pilot \\
\hline Cloudchain & Ramp-up \\
\hline 4Retail & \\
\hline InBitcoin for business & \\
\hline
\end{tabular}

\section{A blockchain architecture continuum}

After having categorized the 19 selected cases according to the features described at the end of paragraph 3, we identified four key architectural 
options: a proprietary ecosystem (1), a semiproprietary ecosystem (2), a proprietary software solution (3) and a fully-decentralized ecosystem (4).

Table 5. Use cases and architectural options

\begin{tabular}{|l|c|c|c|c|}
\hline Blockchain solution & $\mathbf{1}$ & $\mathbf{2}$ & $\mathbf{3}$ & $\mathbf{4}$ \\
\hline The vinicultural supply chain in Italy & & & $\mathrm{x}$ & \\
\hline IoTappo & & & $\mathrm{x}$ & \\
\hline Acincoin & & & $\mathrm{x}$ & \\
\hline Enerchain & & $\mathrm{x}$ & & \\
\hline Maersk: cross-border supply chain solution & $\mathrm{x}$ & & & \\
\hline Hejia: blockchain for pharma procurement & $\mathrm{x}$ & & & \\
\hline Walmart: blockchain for pork meat & $\mathrm{x}$ & & & \\
\hline 4Trace: a blockhain traceability platform & & & & $\mathrm{x}$ \\
\hline SAP - ATB Bank \& Reise Bank AG & & $\mathrm{x}$ & & \\
\hline Timestamping Intesa Sanpaolo & & & & $\mathrm{x}$ \\
\hline That's Mine & & & $\mathrm{x}$ & \\
\hline Ballotchain & & & & $\mathrm{x}$ \\
\hline Smartbond & & & $\mathrm{x}$ & \\
\hline Notarchain & $\mathrm{x}$ & & & \\
\hline Insurechain & & & $\mathrm{x}$ & \\
\hline Securechain & & & $\mathrm{x}$ & \\
\hline Cloudchain & & & $\mathrm{x}$ & \\
\hline 4Retail & $\mathrm{x}$ & & & \\
\hline InBitcoin for business & & & & $\mathrm{x}$ \\
\hline
\end{tabular}

On the basis of this framework, we ended up building a continuum of architectural solutions ranging from a type of IT architecture close to the ones currently deployed by enterprises to one completely decentralized and distributed within the members of a public ecosystem.

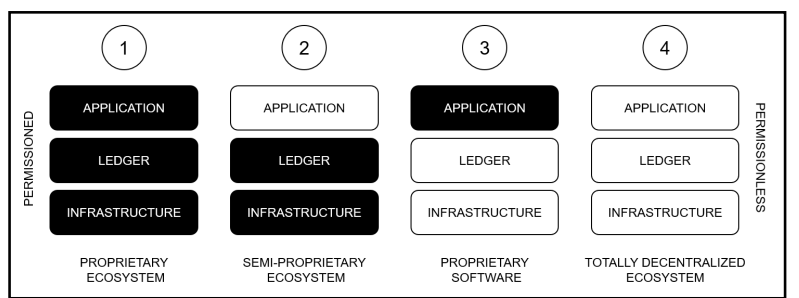

Figure 4. The blockchain architecture continuum

\subsection{Proprietary ecosystem (1)}

In the first blockchain architectural option, the entire ecosystem is internalized within a company or a group of companies (e.g. a consortium) through the development of a whole new blockchain environment, on which the founding member(s) has full and direct control.

5.1.1 Technical Aspects. A company focused on this model needs to develop an entirely proprietary ecosystem. This means that the company will need:
- A dedicated hardware infrastructure aimed at supporting and maintaining the entire ecosystem. The hardware devices will be used both to run the algorithm present in the protocol and to store the information;

- A new blockchain ledger for its own necessities. In that case a company can decide either to develop a new protocol from scratch or to modify an opensource blockchain protocol and adapt it to its needs;

- A dedicated application equipped with an userinterface software, which will be used by each member of the ecosystem to interact with the blockchain ledger.

5.1.2 Governance Aspects. This is a close and fullycontrolled ecosystem. This model is enabled by a private and permissioned blockchain. More precisely:

- Private blockchain. The application is not publicly available. The stakeholders of the entire network are known and there is a decisional authority who controls the users allowed to use the service;

- Permissioned blockchain. The entire ecosystem is maintained by one - or more - central users who verifies each transaction and it is not possible to take part to the consensus mechanism.

5.1.3 Business Aspects. Usually, in such case, the blockchain does not act as a limited software developed for a specific service but assumes the role of an entire new platform in which many different players can take part. In the near future, firms will still exist, and will continue using their legacy systems; however, adding a blockchain ecosystem to the current IT infrastructure could help furtherly improving the levels of the coordination and the transparency along the chain. Thus, this business model does not enable new form of revenues, rather allowing to improve the overall efficiency of an entire, already existing, process.

One of the most relevant examples is that of Notarchain - a private and permissioned platform realized by the Italian notaries. The aim of the Italian notaries was to create a secure, certified and transparent platform to store any kind of digital records (e.g. drawings, works of art, mobile goods) without losing the actual control of their data. Therefore, any information stored is previously checked by an Italian notary who guarantees the truthfulness of the data stored.

\subsection{Semi-proprietary ecosystem (2)}

In the second blockchain option, the company provides part of the ecosystem - i.e. the ledger and the 
infrastructure - allowing authorized third parties to use its blockchain solution in change for some fees.

5.2.1 Technical Aspects. A company can decide to develop a new ledger and offer a dedicated infrastructure to maintain the blockchain as for the first model, but without developing a defined application and user-interface software to interact with it.

5.2.2 Governance Aspects. This is an open but controlled ecosystem. To reach this status, it is required to use a public and permissioned blockchain.

- Public blockchain. The ledger is publicly available. The stakeholders of the entire network are unknown, and they are not required to use a specific software to interact with the underlying ledger and infrastructure;

- Permissioned blockchain. The entire ecosystem is maintained by one (or more) central user who verifies each transaction.

5.2.3 Business aspects. The business implications of this ecosystem strictly depend on the stakeholder we are considering. From one side, there is the blockchain provider, and from the other, the software provider.

- The blockchain provider. The main source of income of the blockchain provider are the fees charged for each transaction occurring in the network (ecosystem fees);

- The software provider. It can either charge some fees (application fees) to the customers who are using the service or just integrate the software within its legacy systems. In this case, the blockchain is used to solve a specific problem related to either transparency, immutability and/or security.

For example, Ripple is working in this way. It is a public permissioned blockchain solution, with a digital currency at its base that enables entities in the ecosystem (especially banks) to send payments across the network. In this case, Ripple provides its own ledger and hardware infrastructure.

If a company wants to use Ripple's blockchain, it must develop its own software to interact with it. SAP developed a blockchain application for cross-border payments between two banks using the Ripple network. The parties involved in the pilot were ATB Financial, one of the largest financial institutions based in Canada, ReiseBank AG, a financial institution based in Germany, SAP and Ripple. In the pilot test, ATB Financial successfully transferred CAD\$1,000 (EUR 667) to ReiseBank using a network built on SAP and Ripple's pioneering network of enterprise blockchain solutions. The payment, which would have typically taken between two to six business days to process, was completed in around 20 seconds.

\subsection{Proprietary software solution (3)}

In the third blockchain architectural option, the company develops a specific software solution in an open blockchain environment. The data (usually encrypted) are freely available to everyone but the final users can access the service only if the provider allows them to.

5.3.1 Technical Aspects In this case, a company does not need to create its own blockchain ledger, nor to dedicate a proprietary hardware infrastructure to create a blockchain-based service. The company simply exploits a totally transparent blockchain ledger, leverage a decentralized hardware infrastructure to build any type of software on top of these two layers.

5.3.2 Governance Aspects In this case, a private application is built on a public and permissionless blockchain. The two main implications are:

- The service - i.e. the blockchain-based application - is not freely available. A company has absolute control on its own solution and can decide to serve a customer at its discretion;

- The blockchain ledger and the infrastructure are totally outsourced. That means that if the ledger shows any type of issues, the company is neither responsible or accountable for that.

These two factors are crucial for a company interested in entering in this world. From one side, being able to totally outsource an entire part of the process is a big opportunity which can lead to costsaving, efficiency and risk-reduction. From the other side, the lack of accountability and responsibility is a threat. A company should be responsible for the correct functioning of its own services.

5.3.3 Business Aspects Companies interested in developing a blockchain-based application would have 3 main benefits:

- Direct business impacts;

- Limited required competences;

- Direct marketing effect for customers.

The major economic benefit for the software provider is represented by the fees it can require to final consumers for using its service. Clearly, a tech company selling "blockchain software" can take advantage of the great momentum surrounding this technology. 
For example, Almaviva, an Italian company leader in the IT sector, has developed a blockchain-based application to notarize and certify the data relative to the Italian vinicultural supply chain. The application is built on the Ethereum blockchain, meaning that all the data, in an encrypted format, are saved on the Ethereum ledger. However, only Almaviva's customers and the Ministry of Agriculture are in possession of the keys required to access the information in a decrypted format.

\subsection{Fully decentralized ecosystem (4)}

In the fourth blockchain architectural option, a company exploits a freely available software solution based on an open blockchain environment. No real developments are required, and the entire blockchain architecture is actually outsourced.

5.4.1 Technical Aspects In this case, a company decides to create a completely decentralized application. As for the third model, the blockchain ledger underlying is public and permissionless. The difference is at a software level. In fact, in this case, the company will create or exploit a:

- A publicly available software;

- A public and permissionless blockchain ledger;

- A decentralized hardware infrastructure.

5.4.2 Governance Aspects In a public ecosystem, anyone can join the network and use the application enabled by the blockchain technology. A user can access a specific service without the authorization of the service provider. In a public and permissionless blockchain, there is no central authority and everyone with an internet connection can use the service (write), read the transaction's history (read) and eventually participate to the consensus mechanism (commit).

5.4.3 Business Aspects A company can either create a new decentralized application or exploit an existing one. The business considerations of these two options are completely different. From one side, a company which decides to create a new decentralized application will benefit from different source of revenues, such as:

- Raised funds: it is likely the company will receive funds from private investors, institutional investors and exchanges. Most of the times, the funds have been raised through an Initial Coin Offering (ICO);

- Application fees: companies can charge some fees to the costumers who are using their blockchain services. Most of the times, to use a fullydecentralized-service, it is required that the final consumer purchases an underlying token associated to that specific service.
From the other side, a company can decide to exploit an already existing application. In this case, the company is not creating a new business model, but rather just adopting an available service. Thus, no new revenue streams are created.

Intesa Sanpaolo, an Italian banking group, prototyped a software solution using the Bitcoin blockchain to notarize financial data and make them available for third parties' investigations. Intesa Sanpaolo holds the databases where all the trading records are saved daily and forwards them via an external timestamp provider (Opentimestamp) to the Bitcoin blockchain.

Thanks to this solution, Intesa Sanpaolo can guarantee to an external auditor the immutability of its trading records. In order to verify that the information have not been tampered with, the auditor can independently check the timestamp recorded in the Bitcoin blockchain.

\subsection{An analytical comparison of the four key blockchain business models}

As described in the previous paragraph, different blockchain business models widely differ between each other. Table 5 provides a high-level comparison of the architectural options considered.

\begin{tabular}{|c|c|c|c|c|c|}
\hline & \multicolumn{4}{|c|}{ Blockchain Architecture Continuum } \\
\hline & & 1 & 2 & 3 & 4 \\
\hline \multirow{3}{*}{ 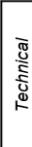 } & $\begin{array}{c}\text { Application } \\
\text { layer }\end{array}$ & Proprietary & Third Party & Proprietary & Third Party \\
\hline & $\begin{array}{l}\text { Ledger } \\
\text { layer }\end{array}$ & Proprietary & Proprietary & Third Party & Third Party \\
\hline & $\begin{array}{c}\text { Hardware } \\
\text { layer }\end{array}$ & Proprietary & Proprietary & Third Party & Third Party \\
\hline 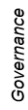 & $\begin{array}{c}\text { Type of } \\
\text { ownership } \\
\text { and } \\
\text { governance }\end{array}$ & $\begin{array}{l}\text { Private and } \\
\text { permissioned }\end{array}$ & $\begin{array}{l}\text { Public and } \\
\text { permissioned }\end{array}$ & $\begin{array}{c}\text { Private and } \\
\text { permissionless }\end{array}$ & $\begin{array}{l}\text { Public and } \\
\text { permissionless }\end{array}$ \\
\hline 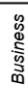 & $\begin{array}{l}\text { Revenue } \\
\text { models }\end{array}$ & $\begin{array}{c}\text { No new revenues } \\
\text { stream }\end{array}$ & $\begin{array}{l}\text { Ecosystem } \\
\text { fees }\end{array}$ & $\begin{array}{c}\text { Application } \\
\text { fees }\end{array}$ & $\begin{array}{l}\text { Raised funds; } \\
\text { Application } \\
\text { fees }\end{array}$ \\
\hline
\end{tabular}

Figure 5. A comparison of blockchain architectural models

\section{Conclusion}

The IS Research should play a leading role in facilitating the transition from a post-hype phase to value driven applications of blockchain technologies. In order to meet this goal and create a valuable contribution to the business community, scholars should leverage on a common language and approach to structure their research effort. According to this perspective, we started from the most relevant pieces of academic literature addressing the description of a blockchain architecture. Although it is commonly accepted that any blockchain could be represented as a 
layered architecture, none of the analysed studies introduces a dynamic view of the architectural options that could be designed starting from the static representation of the technical layers. Moreover, none of the current studies addresses non-technical architectural dimensions such as the governance model of the technical layers, as well as the business model that could be built on top of the blockchain.

Starting from the static view of a blockchain solution, we created a comprehensive continuum of architectural options ranging from the fully proprietary solution - where all the technical layers are owned by a company or a company's ecosystem or even a private consortium - to the fully decentralized solution where all the technical layers rely on public available blockchain protocols such as the Bitcoin's one.

Backed by the qualitative observation of relevant and functioning blockchain projects, we described each of the four emergent architectural archetypes, adding some considerations in terms of the currently implemented business models.

Our work facilitates the comprehension of the blockchain technology from various points of view, especially considering the governance, ownership and business model perspectives. At a governance level, we can affirm that blockchain is a technology which enables different business models starting from the ones that we know today, based on the paradigm of centralization and third-party intermediaries. In particular, business models built on blockchain could represent a way towards a more balanced distribution of power between companies and consumers. This is especially true the more we move away from the fully proprietary solution, that basically tends to replicate established and well-known business models.

It must be always remembered that blockchain makes little sense if considered in terms of a proprietary technology: what gives value to this new paradigm is the construction of a network that revolves around it and exploits it jointly. The pervasive nature of this new technology could effectively make it the technology behind the keeping of any type of registry, providing the three main features that blockchain enables: transparency, immutability and security. Moreover, the open nature of most blockchain protocols enables a certain degree of interoperability between them, which in the future could lead to the use of blockchain as a technology shared by the various components of an ecosystem. On the other side, from an economic/strategic point of view, the comprehensive architecture continuum we have developed can represent an ideal starting point, especially for those working in an enterprise environment, to evaluate if there is a real and tangible sense in implementing a blockchain solution in their companies. The continuum provides a useful framework to determine whether or not the deployment of blockchain solution fits in a certain context, and above all if its introduction can represent a source of added value for the company. In fact, it is likely that precisely because of its original "anarchic" nature, very far from current business realities (even the most advanced in the IT field), a blockchain solution could rather represent an element of negative value if implemented without having deeply understood the transformation and the challenges that it brings from a governance and business model perspective.

It is worth noting that the architecture continuum herewith presented could be used by business and IT executives in order to develop an implementation roadmap to support their blockchain strategy. Since the 4 architectural options are not mutually exclusive, a company would define its current positioning and design an evolution strategy from a fully proprietary model towards a fully decentralized one, as well as segment its processes and activities and find the most coherent model on the continuum.

From a research perspective, the continuum proposed in this work could represent a starting point for furtherly investigating the implementation of blockchain technologies. First, the framework could be broadened with additional variables, as they will emerge from the literature. Second, it could be applied to a wider sample of case studies in order to verify the coherence between technical and governance decisions.

\section{References}

[1] B. Carson, G. Romanelli, P. Walsh, A. Zhumaev, "Blockchain beyond the hype: What is the strategic business value?”. McKinsey \& Company, June 2018.

[2] A. Martin, "Davos: Blockchain can no longer be ignored”. Financial Times, January 24, 2018.

[3] R. Hackett, "Why Big Business Is Racing to Build Blockchains". Fortune Magazine, August 22, 2017.

[4] A. Lyall, P. Mercier, S. Gstettner, "The Death of Supply Chain Management". Harvard Business Review, June 15, 2018.

[5] M. Casey, P. Wong, "Global supply chains are about to get better, thanks to blockchain". Harvard Business Review, March 13, 2017.

[6] G. Salviotti, L. De Rossi, N. Abbatemarco "A structured framework to assess the business application landscape of blockchain technologies" in The 51st Hawaii International Conference on System Sciences, 2018.

[7] M. Friedlmaier, A. Tumasjan, I.M. Welpe, "Disrupting Industries With Blockchain: The Industry, Venture Capital Funding, and Regional Distribution of Blockchain Ventures”, SSRN 2854756, 2016. 
[8] X. Xu, I. Weber, M. Staples, L. Zhu, J. Bosch, L. Bass, P. Rimba. "A taxonomy of blockchain-based systems for architecture design". In International Conference on Software Architecture (pp. 243-252), 2017.

[9] M. Morini, "From Blockchain Hype to a Real Business Case for Financial Markets". 2016.

[10] B. Notheisen, F. Hawlitschek and C. Weinhardt, "Breaking Down the Blockchain Hype-Towards a Blockchain Market Engineering Approach.”, 2017.

[11] M. Iansiti and K. R. Lakhani, "The truth about blockchain.”, Harvard Business Review, vol. 95.1, pp. 118-127, 2017.

[12] J. Mattila, "The Blockchain Phenomenon-The Disruptive Potential of Distributed Consensus Architectures", The Research Institute of the Finnish Economy, 2016.

[13] D. Tapscott, A. Tapscott, "Blockchain Revolution: How the Technology Behind Bitcoin Is Changing Money, Business, and the World". Penguin, 2016

[14] C. Mulligan, J. Z. Scott, S. Warren, JP Rangaswami, "Blockchain Beyond the Hype - A Practical Framework for Business Leaders". WEF White Paper, April 2018.

[15] K. Panetta, "Top 10 Mistakes in Enterprise Blockchain Projects". Gartner, Feb 2017.

[16] Z. Zheng, S. Xie, H. N. Dai, H. Wang, "Blockchain challenges and opportunities: A survey". 2016.

[17] A. Deshpande, K. Stewart, L. Lepetit, S. Gunashekar, "Distributed Ledger Technologies/Blockchain: Challenges, opportunities and the prospects for standards". The British Standards Institution, 2017.

[18] D. Furlonger, R. Kandaswamy, "Blockchain Status 2018: Market Adoption Reality”. March 2018.

[19] V. Morabito, "Business Innovation Through Blockchain.”, Springer International Publishing, 2017.

[20] F. Glaser, "Pervasive decentralisation of digital infrastructures: a framework for blockchain enabled system and use case analysis." 2017.

[21] P. Tasca, T. Thanabalasingham and T. Claudio J., "Ontology of Blockchain Technologies. Principles of identification and classification", 2017.

[22] J. de Kruijff and H. Weigand, "Understanding the blockchain using enterprise ontology.," in International Conference on Advanced Information Systems Engineering, 2017.

[23] K. M. Eisenhardt and M. E. Graebner, "Theory building from cases: Opportunities and challenges." Academy of management journal, vol. 50.1, pp. 25-32, 2007.

[24] M. Walport, "Distributed ledger technology: Beyond blockchain.”, UK Government Office for Science, 2016.

[25] A. Narayanan, J. Bonneau, E. Felten, A. Miller, S. Goldfeder, "Bitcoin and cryptocurrency technologies: a comprehensive introduction". Princeton University Press, 2016.

[26] A. Baliga, "Understanding Blockchain Consensus Models", April 2017.
[27] M. Swan, "Blockchain. Blueprint for a new Economy", O'Really Media, 2015.

[28] R. Beck, M. Avital, M. Rossi and J. B. \& Thatcher, Blockchain Technology in Business and Information Systems Research., 2017. Information Systems.

[29] J. T. Lindman, "Opportunities and risks of Blockchain Technologies-a research agenda." 2017.

[30] G. Hileman and M. Rauchs, "Global blockchain benchmarking study.", 2017.

[31] I. Benbasat, "Empirical research in information systems: the practice of relevance". MIS quarterly, 1999.

[32] O. N. Babüroglu \& I. Ravn, "Normative action research. Organization Studies," vol. 13.1, pp. 19-34, 1992.

[33] R. Baskerville and M. D. Myers, "Special issue on action research in information systems: Making IS research relevant to practice: Foreword." MIS quarterly, pp. 329-335, 2004.

[34] M. Uschold and M. Gruninger, "Ontologies: Principles, methods and applications.", The knowledge engineering review, vol. 11, no. 2, pp. 93-136., 1996.

[35] S. Gregor, "The nature of theory in information systems.," MIS quarterly, pp. 611-642, 2006.

[36] A. Tiwana, B. Konsynski and A. A. Bush, "Research commentary-Platform evolution: Coevolution of platform architecture, governance, and environmental dynamics." Information systems research, vol. 21 , no. 4 , pp. 675-687, 2010.

[37] K. Ulrich, "The role of product architecture in the manufacturing firm." Research policy, vol. 24.3, pp. 419-440, 1995.

[38] B. Kaplan and J. A. Maxwell, "Evaluating health care information systems: Methods and applications. Qualitative Research Methods for Evaluating Computer Information Systems. JG Anderson, CE Ayden and SJ Jay.", Thousand Oaks, Sage., 1994.

[39] M. D. Myers, "Qualitative research in information systems.", Management Information Systems Quarterly, vol. 21, no. 2, pp. 241-242, 1997.

[40] I. Benbasat, D. K. Goldstein and M. Mead, "The case research strategy in studies of information systems." MIS quarterly, pp. 369-386, 1987.

[41] R. H. Hayes, S. C. Wheelwright, K. B. Clark, "Dynamic manufacturing: Creating the learning organization". Simon and Schuster, 1988.

[42] K. M. Eisenhardt, "Better stories and better constructs: The case for rigor and comparative logic." Academy of Management review, vol. 16, no. 3, pp. 620-627, 1991.

[43] R. Yin, "Case study research: Design and Methods, Applied social research methods series, band 5, Applied social research methods series", 1994.

[44] S. L. Brown and K. M. Eisenhardt, "The art of continuous change: Linking complexity theory and time-paced evolution in relentlessly shifting organizations.," Administrative science quarterly, pp. 134, 1997. 